IMA Journal of Applied Mathematics (2006) 71, 359-382

doi:10.1093/imamat/hxh100

Advance Access publication on September 16, 2005

\title{
On a modified version of ILDM approach: asymptotic analysis based on integral manifolds
}

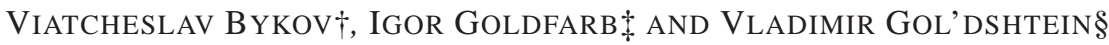 \\ Ben-Gurion University of the Negev, PO Box 653, Beer-Sheva 84105, Israel \\ AND \\ ULRICH MAASTI \\ Institute for Technical Thermodynamics, Karlsruhe University (TH), \\ Kaiserstrasse 12, D76149 Karlsruhe, Germany
}

[Received on 22 January 2004; accepted on 16 June 2005]

\begin{abstract}
Using the method of integral (invariant) manifolds, the intrinsic low-dimensional manifolds (ILDM) method is analysed. This is a method for identifying invariant manifolds of a system's slow dynamics and has proven to be an efficient tool in modelling of laminar and turbulent combustion. It allows treating multi-scale systems by revealing their hidden hierarchy and decomposing the system dynamics into fast and slow motions. The performed analysis shows that the original ILDM technique can be interpreted as one of the many possible realizations of the general framework, which is based on a special transformation of the original coordinates in the state space. A modification of the ILDM is proposed based on a new definition of the transformation matrix. The proposed numerical procedure is demonstrated on linear examples and highly non-linear test problems of mathematical theory of combustion and demonstrates in some cases better performance with respect to the existing one.
\end{abstract}

Keywords: reduction; integral manifolds; singularly perturbed system; decomposition; ILDM.

\section{Introduction}

Mathematical models of chemical, biochemical and mechanical systems are often formulated as a large set of differential equations, and for the purpose of numerical and/or analytical analysis, it is often desirable to reduce to a smaller system without essential loss of accuracy. Experience witnesses that a large set of differential equations describing complex chemical or mechanical phenomenon generally has a number of different time-scales of subprocesses. This hierarchical structure allows to apply various asymptotic approaches for analysis of the system dynamics. In the geometrical version of the interpretation of the dynamics, one can describe the multi-scale hierarchy as a progressive motion through surfaces of lower and lower dimensionality (these surfaces are called invariant manifolds). At present, there are a number of asymptotic tools able to treat the multi-scale system of equations and to expose a 'hidden' hierarchy of the original system of governing equations. An incomplete list includes computational singular perturbation method (Hadjinicolaou \& Goussis, 1999; Lam \& Goussis, 1988, 1994; Valorani \& Goussis, 2001), method of integral manifolds (MIM) (Fenichel, 1979; Gol'dshtein \&

\footnotetext{
${ }^{\dagger}$ Email: vbykov@cs.bgu.ac.il

\$Email: goldfarb@cs.bgu.ac.il

§Email: vladimir@bgumail.bgu.ac.il

IEmail: umaas@itt.mach.uni-karlsruhe.de
}

(c) The Author 2005. Published by Oxford University Press on behalf of the Institute of Mathematics and its Applications. All rights reserved. 
Sobolev, 1988, 1992), and intrinsic low-dimensional manifolds (ILDM) (Maas \& Pope, 1992; Rhodes et al., 1999; Kaper \& Kaper, 2001).

The numeric asymptotic method (ILDM), which is particularly in the focus of our analysis in the present piece of work, was developed originally for the investigation of the combustion phenomena. Normally, a mathematical model for gas-phase or multi-phase chemical reaction systems consists of a large set of partial differential equations, which describe the time-dependent development of all the properties that determine the state of the system. The governing processes (i.e. flow, molecular transport and chemical reactions) occur at time-scales that differ by orders of magnitude. A presence of the different time-scales is the reason that the system of that type has an internal hierarchy. The governing ratio behind the original algorithm of ILDM may be interpreted as a global transformation of the original coordinates aimed to decompose processes with essentially different rates of changes. Local-dependent relations between the original variables serve as a basis of the decomposition.

The investigation of the existing version of the ILDM technique is performed from the point of view of some general framework based on a special transformation of the original coordinates in the phase space (variables), so that the new introduced coordinates represent a union of the two separate setsfast and slow ones. It is shown that the ILDM technique de facto represents one of the many possible realizations of the general framework. As any other method of analysis, ILDM has its own restrictions and drawbacks. A detailed analysis of some of these restrictions allows to suggest a modification of the existing version of the ILDM technique, which is based on another definition of the transformation matrix and can be conditionally called TILDM. Application of the suggested TILDM technique demonstrates that the uncovered restrictions of the ILDM method are overcome. Moreover, it turns out that the suggested approach becomes effective in situations, which were not expected (in particular, in highly non-linear problems of mathematical theory of combustion).

The outline of this paper is as follows. Section 2 starts with background information useful for the rest of the article, namely, general description of the so-called singular perturbed system (SPS) of ordinary differential equations (ODEs). Following a description of the basic properties of the SPS of ODEs, we expose the possible multiple scales character of an arbitrary system (internal hierarchy), which is generally 'hidden' within the system. A simple example of transformation of coordinates, which allows to re-write the original multi-scale system in the conventional form of the SPS of ODEs, is presented. Following the example, a general framework of determination of the fast and slow variables by an introduction of the new coordinates is formulated. A brief description of the ILDM approach in the light of the general framework based on the subdivision of the fast and slow variables closes the Section 2. Section 3 deals with a number of artificial examples, which illustrate restrictions of the existing version of the ILDM approach. Section 4 suggests a modification of the existing version of the ILDM approach. It starts from the discussion concerning the geometrical sense of the linear transform of the original coordinates and continues with a formulation of the suggested TILDM approach. Further, an asymptotic analysis of the suggested approach in the 2D case is presented. In Section 5, we present a number of examples, which are treated by the suggested approach (TILDM), and demonstrate the advantages of the proposed technique. Finally, we summarize our conclusions regarding relative advantages and disadvantages of the suggested approach and the possible ways of further investigations of its properties (Section 6).

\section{Theoretical background}

Many physical, biological, chemical and mechanical systems are characterized by a large amount of unknowns and a wide range of temporal or spatial scales. These scales govern the underlying differential equations and create serious difficulties (stiffness) for solution proceeding, no matter which 
approach — analytical or numerical - is chosen. In order to address the issues of both stiffness and physical understanding, a variety of asymptotic tools were developed, which explore the existing gap in scales.

Most theoretical asymptotic tools deal with the systems written in the specific form of SPS of ODEs. The distinguishing feature of this type of systems is the explicit determination of the singular parameter (small parameter(s) of the considered physical system). To give a reader a bit more detailed description of the SPS, let us give here a short description of this type of system of ODEs.

\section{$2.1 \quad$ Singularly perturbed systems}

Singularly perturbed systems (SPSs) are systems containing ODEs, which exhibit dynamic behaviour evolving two (or more) vastly different time-scales. The conventional form of the SPS consists of a set of equations in which derivatives of a number of variables are multiplied by a small parameter

$$
\begin{aligned}
\varepsilon \frac{\mathrm{d} \vec{X}}{\mathrm{~d} t} & =F(\vec{X}, \vec{Y}, \varepsilon), \\
\frac{\mathrm{d} \vec{Y}}{\mathrm{~d} t} & =G(\vec{X}, \vec{Y}, \varepsilon) .
\end{aligned}
$$

It is worthwhile to mention here that the presentation of the original system in the form of the set (2.1.1)-(2.1.2) is a simplification to some extent. The general problem is normally harder than that described by the system (2.1.1)-(2.1.2) due to a possible presence of a large number of different time-scales (the set (2.1.1)-(2.1.2) describes the pair of them only) and an absence of the explicit small parameter(s). However, the simplification made above does not effect the nature of the performed analysis.

When the parameter $\varepsilon$ is small with respect to unity and the functions in the right-hand side (RHS) of (2.1.1)-(2.1.2) are of the same order $G(\vec{X}, \vec{Y}, \varepsilon) / F(\vec{X}, \vec{Y}, \varepsilon) \propto \mathrm{O}(1)$, the system (2.1.1)-(2.1.2) exhibits a dynamical behaviour characterized by a presence of two sufficiently different time-scales. The difference between rates of changes of the two vectors $(G(\vec{X}, \vec{Y}, \varepsilon), F(\vec{X}, \vec{Y}, \varepsilon))$ is determined by the small parameter $\varepsilon$. For our further convenience in the present paper, we will suppose that both functions in the RHS of (2.1.1)-(2.1.2) are sufficiently smooth (infinitely differentiable). Additionally, we assume that the RHS of the equations do not contain a time-like variable $t$ in the explicit form (we will restrict ourselves to autonomous systems only).

Note here that a rate of change of the vector $\vec{X}$ tends to infinity when $\varepsilon \rightarrow 0$ (assume that $F(\vec{X}, \vec{Y}, \varepsilon) \neq 0)$. According to this elementary analysis of (2.1.1)-(2.1.2), the first of the two equations is called fast subsystem, while the second is called slow subsystem.

Theoretical problems arising from the system (2.1.1)-(2.1.2) are elaborated rather well in the general theory of integral (invariant) manifolds, which was developed for non-linear mechanics in a number of works (Bogolyubov \& Mitropolsky, 1961; Fenichel, 1979; Hale, 1969; Mitropolskiy \& Lykova, 1968; Strygin \& Sobolev, 1988). The theory states that the system (2.1.1)-(2.1.2) has a unique integral (invariant) manifold that can be represented as a series with respect to the small parameter $\varepsilon$. The advantage of the manifold's existence is the fact that the analysis of the system behaviour can be considerably simplified by reducing the dimension of the system to the dimension of the slow variables. In $\mathrm{O}(\varepsilon)$ approximation of the slow invariant manifold, the analysis of the original system can be reduced to the analysis on the slow manifold. The determination of the exact form and location of the slow manifold is normally a rather complex problem, which can eliminate the advantage of the dimension reduction. 
Fortunately, the zeroth approximation of the exact slow manifold can be found relatively simply just by equating the RHS of the fast equation (2.1.1) to zero. The obtained equation determines the so-called slow surface (curve in the 2D case) and the analysis of the original system can be reduced to the analysis on the slow surface.

On the slow surface, the changes of the slow and fast variables are comparable (i.e. fast and slow processes are balanced). Beyond the slow surface, the slow variables are asymptotically constant (quasistationary-in an $\mathrm{O}(\varepsilon)$ approximation). Therefore, a trajectory of the system in the phase space can be naturally decomposed into 'fast' parts (which are off the slow manifold) and 'slow' parts (which are on the slow manifold). The 'fast' and 'slow' parts of a trajectory can follow each other. The main types of system trajectories can be predicted by the use of the slow surface. This technique of the asymptotic analysis, which got the abbreviation MIM (method of integral (invariant) manifolds), was adopted for problems of chemical kinetics (Davis \& Skodje, 1999) and combustion by Gol'dshtein \& Sobolev (1988). It has been developed for studying unravelling problems of gaseous combustion (Gol'dshtein \& Sobolev, 1988), catalysis (Babushok \& Gol'dshtein, 1988; Gol'dshtein \& Sobolev, 1992), non-linear theory of control (Strygin \& Sobolev, 1988), self-ignition in multi-phase media (Gol'dshtein et al., 1996; Goldfarb et al., 1996, 1997, 1998; McIntosh et al., 1988; Bykov et al., 2002) and flame propagation in porous media (Goldfarb et al., 1999).

\subsection{Internal 'hidden' hierarchy}

In most cases, the system of the governing equations looks like the system (2.1.1)-(2.1.2) in a rich imagination of a pure theoretician only. In most of the engineering problems of actual importance, the original system of governing equations contains its own internal hierarchy (i.e. a small parameter of the considered physical system), but it is presented implicitly. To re-write the original system in the conventional form of the SPS (2.1.1)-(2.1.2) sometimes demands non-trivial efforts. The most popular situation is when a researcher is required to investigate a system of governing equations in the general form (splitting of the rates of change of various processes involved exists, but it is not presented as small parameter in the explicit form). In other words, the internal hierarchy of the system is hidden. Two natural questions arise-whether it is possible (1) to discover an existence of and (2) to expose the unclosed hierarchy? In the language of the present paper-whether are we able to subdivide the processes (or their combinations) into the slow and the fast ones on the basis of the analysis of the original system of ODEs?

To illustrate the idea, consider the Michaelis-Menten mechanism, which is the basic building block for enzymological modelling (Laidler \& Bunting, 1973). The set of governing equations for one of realistic cases (a reader is referred to Laidler \& Bunting, 1973, Roussel, 1997 and Roussel \& Fraser, 2001 for more details) may be presented as following 2D system of ODEs

$$
\left\{\begin{array}{llrl}
\frac{\mathrm{d} X}{\mathrm{~d} t} & =-(X(1-Y)-Y)+\varepsilon Y, & & \varepsilon \ll 1, \\
\frac{\mathrm{d} Y}{\mathrm{~d} t}=\beta(X(1-Y)-Y), & & \beta \sim \mathrm{O}(1),
\end{array}\right.
$$

where $X$ is the dimensionless concentration of the reactant (substrate), $Y$ is the dimensionless concentration of the enzyme-substrate complex and $\varepsilon$ and $\beta$ are parameters related to the rates of reactions involved.

The form of the original system (2.2.1) does not allow to immediately suspect an existence of the internal hierarchy in the system (2.2.1), and, in turn, a possibility to extract a small parameter, which could correspond to the hierarchy. Nevertheless, a presence of the small parameter $\varepsilon$ hints that 
there is a 'hidden' hierarchy in the system (2.2.1). Indeed, introducing a pair of the new auxiliary coordinates

$$
u=\beta X+Y, \quad v=-X+\beta Y
$$

and substituting $\tau=t \varepsilon$, we can re-write the original system (2.2.1) in the form

$$
\left\{\begin{array}{l}
\frac{\mathrm{d} u}{\mathrm{~d} \tau}=\beta Y(u, v)=F(u, v), \\
\varepsilon \frac{\mathrm{d} v}{\mathrm{~d} \tau}=\left(1+\beta^{2}\right)(X(u, v)(1-Y(u, v))-Y(u, v))-\varepsilon Y(u, v)=G(u, v) .
\end{array}\right.
$$

In another words, introducing a pair of the variables brings the system to the conventional form of the SPS (slow variable $u$ and fast one $v$ ). Note here that the substitution (2.2.2) is almost a rotation (due to the evident fact $\beta \sim \mathrm{O}(1)$ ), a clear hierarchy exists.

As a result of the analysis of this simple example, one can conclude that there are at least some specific cases, when an elementary linear transformation of the original coordinates (similar to (2.2.2)) solves a problem of a decomposition of the original mixed dynamics into the two separate motions-fast and slow. Just after an application of the linear transformation, the introduced coordinates $(u, v)$ become the directions of the chosen types of motion (fast and slow).

The Michaelis-Menten mechanism considered above demonstrates that a linear transformation of the type (2.2.2) allows us to disclose the 'hidden' hierarchy of the original system (2.2.1). It is of interest to develop a technique, which would be able (1) to determine whether a given system of ODEs possesses an internal hierarchy and (2) to provide us with a simple way of finding a desired transformation. It would be natural to suppose that there is more general transformation of the original coordinates, which allows us to gain the declared purposes. This generalization of the example presented here is described in the following section.

\subsection{General framework—subdivision into 'fast' and 'slow' variables}

This section focuses on a general-type decomposition of an arbitrary vector field into fast and slow subfields in the spirit of approach used in the previous section.

As we could see in the previous section, it is possible to find a suitable transformation of the original coordinates, which provides us with the desired result-in the new coordinates, the fast and the slow motions are decomposed and are performed along the new introduced axes. It is of interest to elucidate whether the adopted approach of coordinate transformation is a universal one.

To formalize the question, consider an arbitrary system of ODEs of the type

$$
\frac{\mathrm{d} Z}{\mathrm{~d} t}=\Phi(Z), \quad Z \in \mathbb{R}^{n} .
$$

Reduction of the original problem (2.3.1) to the conventional SPS form (it may be understood to some extent as vector field decomposition) may be formalized according to the following procedure. Consider a decomposition of the type $n \rightarrow n=n_{\mathrm{f}}+n_{\mathrm{s}}$, where the subscripts $\mathrm{f}$ and s relate to the fast and slow new variables, respectively. Suppose that there exists a smooth matrix $\widetilde{Q}(Z)=$ $\left(\begin{array}{c}\widetilde{Q}_{\mathrm{f}}(Z) \\ \widetilde{Q}_{\mathrm{s}}(Z)\end{array}\right)$, det $\widetilde{Q}(Z) \neq 0\left(\widetilde{Q}_{\mathrm{f}}\right.$ is an $n_{\mathrm{f}} \times n$ matrix and $\widetilde{Q}_{\mathrm{s}}$ is an $n_{\mathrm{s}} \times n$ matrix$)$, such that the matrix $\widetilde{Q}$ produces a desired decomposition of the original variables of the system (2.3.1) into the two groups of the fast and slow new variables

$$
W=\left(\begin{array}{c}
U \\
V
\end{array}\right)=\widetilde{Q} Z=\left(\begin{array}{c}
\widetilde{Q}_{\mathrm{f}}(Z) \\
\widetilde{Q}_{\mathrm{s}}(Z)
\end{array}\right) Z, \quad U=\widetilde{Q}_{\mathrm{f}}(Z) Z=\left(\begin{array}{c}
u_{1} \\
\vdots \\
u_{n_{\mathrm{f}}}
\end{array}\right), \quad V=\widetilde{Q}_{\mathrm{s}}(Z) Z=\left(\begin{array}{c}
v_{1} \\
\vdots \\
v_{n_{\mathrm{s}}}
\end{array}\right) .
$$


Now, re-write the system (2.3.1) in the new introduced variables

$$
\begin{aligned}
& \frac{\mathrm{d} U}{\mathrm{~d} t}=\frac{\mathrm{d} \widetilde{Q}_{\mathrm{f}}(Z)}{\mathrm{d} t} Z+\widetilde{Q}_{\mathrm{f}}(Z) \frac{\mathrm{d} Z}{\mathrm{~d} t}=\frac{\mathrm{d} \widetilde{Q}_{\mathrm{f}}(Z)}{\mathrm{d} t} Z+\widetilde{Q}_{\mathrm{f}}(Z) \Phi(Z), \\
& \frac{\mathrm{d} V}{\mathrm{~d} t}=\frac{\mathrm{d} \widetilde{Q}_{\mathrm{s}}(Z)}{\mathrm{d} t} Z+\widetilde{Q}_{\mathrm{s}}(Z) \frac{\mathrm{d} Z}{\mathrm{~d} t}=\frac{\mathrm{d} \widetilde{Q}_{\mathrm{s}}(Z)}{\mathrm{d} t} Z+\widetilde{Q}_{\mathrm{s}}(Z) \Phi(Z) .
\end{aligned}
$$

RHS of the last two equations is written for some solution $Z=Z(W)$ of the equation $W=\widetilde{Q}(Z) Z$, i.e. for this solution we can simply write

$$
\begin{array}{ll}
\frac{\mathrm{d} U}{\mathrm{~d} t}=\frac{\mathrm{d} \widetilde{Q}_{\mathrm{f}}(W)}{\mathrm{d} t} Z(W)+\Phi_{\mathrm{f}}(W), & \Phi_{\mathrm{f}}(W)=\widetilde{Q}_{\mathrm{f}}(Z) \Phi(Z), \\
\frac{\mathrm{d} V}{\mathrm{~d} t}=\frac{\mathrm{d} \widetilde{Q}_{\mathrm{s}}(W)}{\mathrm{d} t} Z(W)+\Phi_{\mathrm{s}}(W), & \Phi_{\mathrm{s}}(W)=\widetilde{Q}_{\mathrm{s}}(Z) \Phi(Z) .
\end{array}
$$

According to our assumption, the part $U$ of the new variable $W$ should be fast, whereas the part $V$ should be slow. It means that each component of the fast part of the vector field (determined by the RHS of (2.3.5)) must be essentially larger than any other component of its slow counterpart

$$
\begin{aligned}
\left|\frac{\mathrm{d} u_{i}}{\mathrm{~d} t}\right| & \gg\left|\frac{\mathrm{d} v_{j}}{\mathrm{~d} t}\right|, \quad \forall i=1, \ldots, n_{\mathrm{f}}, \quad \forall j=1, \ldots, n_{\mathrm{s}}, \\
& \Rightarrow\left|\left(\frac{\mathrm{d} \widetilde{Q}_{\mathrm{f}}(W)}{\mathrm{d} t} Z(W)+\Phi_{\mathrm{f}}(W)\right)_{i}\right| \gg\left|\left(\frac{\mathrm{d} \widetilde{Q}_{\mathrm{s}}(W)}{\mathrm{d} t} Z(W)+\Phi_{\mathrm{s}}(W)\right)_{j}\right| .
\end{aligned}
$$

On the other hand, having in mind a clear presumption that the dynamics of the system is governed by the vector field $(\Phi)$ and not by the transformation matrix $\widetilde{Q}$, one can formulate an additional restriction for the matrix $\widetilde{Q}$ : it should be almost constant. More correctly, the following relations should be valid

$$
\left|\left(\frac{\mathrm{d} \widetilde{Q}_{\mathrm{f}}(W)}{\mathrm{d} t} Z(W)\right)_{i}\right| \ll\left|\left(\Phi_{\mathrm{f}}(W)\right)_{i}\right|, \quad \forall i=1, \ldots, n_{\mathrm{f}} .
$$

Once the conditions (2.3.7) and (2.3.8) are fulfilled, the transformation of the original system (2.3.1) reaches its aim and the derived system (2.3.5)-(2.3.6) actually represents the SPS. As a result, the general theory of the invariant manifolds is applicable. Under the aforementioned requirements, the fast subsystem of the system (2.3.5)-(2.3.6) can be re-written in the simpler form

$$
\frac{\mathrm{d} U}{\mathrm{~d} t} \approx \Phi_{\mathrm{f}}(W)
$$

which allows us to determine a location of the manifold (more correctly, its approximation). Recalling here that the zeroth approximation of the exact slow manifold can be determined by equating the RHS of the fast subsystem to zero. In accepted terms, this can be re-written as $\Phi_{\mathrm{f}}(W)=0$, which gives us the definition of the slow manifolds in the framework of the suggested transformation of the original coordinates in the form

$$
\Phi_{\mathrm{f}}(W)=\widetilde{Q}_{\mathrm{f}}(Z) \Phi(Z)=0 .
$$

To perform reduction in such a way, we need a special machinery that can permit to construct fast-slow decompositions of vector fields. In other words, we need to build an algorithm allowing to 
determine the transformation matrix $\widetilde{Q}$. It is clear that the prospective algorithm should contain some necessary steps, which can be formulated as follows: (a) evaluation of dimensions of fast and slow subfields on the basis of the analysis of the original vector field $(\Phi(Z))$, (b) construction of the transformation matrix $\widetilde{Q}$ for transition from the original variables $Z$ to the new variables $W$ and (c) verification of whether the conditions (2.3.7) and (2.3.8) of the algorithm are fulfilled.

The ILDM procedure of the decomposition of the original system of ODEs, which will be briefly described in the next section, can be considered as one of the numerical algorithms built according to the general approach discussed in this section.

\subsection{Method of ILDM (Maas \& Pope, 1992)}

Let us describe here very briefly the essential steps of the ILDM in the light of the general framework discussed in the previous section. Assume that the system of ODEs of the type (2.3.1) can be represented locally as a multi-scale system for a corresponding choice of a local basis. The last depends on the choice of an arbitrary point $Z$ in the $n$-dimensional Euclidian space $\mathbb{R}^{n}$. It means that in this local basis, a separation of variables in accordance with their rates of changes is possible (i.e. the considered system can be re-written in this local basis for some neighbourhood of the point $Z$ as SPS of ODEs). According to the assumption, the system can be subdivided locally into fast relaxing and slow or non-relaxing subsystems. Suppose that the fast subsystem has the same dimension $n_{\mathrm{f}}\left(n_{\mathrm{f}}<n\right)$ at any point $Z \subseteq \mathbb{R}^{n}$.

For typical situations, a set of all steady states of the fast subsystem represents an $n_{\mathrm{s}}$-dimensional slow manifold $\left(n_{\mathrm{s}}=n-n_{\mathrm{f}}\right.$ ) and our aim is to determine its location. The authors of ILDM suggested that the dynamics of the overall system from arbitrary initial condition should decay very quickly onto this $n_{\mathrm{s}}$-dimensional manifold. The ILDM allows to identify approximately (as a set of separate points) the slow invariant manifolds (the so-called ILDM).

These manifolds can be found in the following manner (Maas \& Pope, 1992 and Maas, 1994). Suppose a local basis of the original phase space is formed by the invariant subspaces of the Jacobi matrix $M_{\mathrm{J}}$ (Jacobian) of the vector field $\Phi$ at an arbitrary point $Z_{0}$. If the set of eigenvalues $\lambda_{i}$ can be subdivided into two groups

$$
\max \left\{\operatorname{Re}\left[\lambda_{i}\right], i=1, \ldots, n_{\mathrm{f}}\right\} \ll \tau<\min \left\{\operatorname{Re}\left[\lambda_{i}\right], i=n_{\mathrm{f}}+1, \ldots, n\right\}, \quad \tau<0,
$$

one can introduce invariant subspaces $T_{\mathrm{f}}$ and $T_{\mathrm{s}}$. $T_{\mathrm{f}}$ is spanned by the eigenvectors corresponding to the eigenvalues with large negative (fast) real parts. In turn, the subspace $T_{\mathrm{s}}$ is spanned by the eigenvectors corresponding to the eigenvalues with small negative or positive (slow) real parts. Therefore, the transition matrix $Q(Z)$ from this local basis to the standard one that is constructed from the eigenvectors of the Jacobi matrix and its inverse $Q^{-1}(Z)$ can read like two block matrices

$$
Q=\left(\begin{array}{ll}
Q_{\mathrm{f}} & Q_{\mathrm{s}}
\end{array}\right), \quad Q^{-1}=\left(\begin{array}{c}
\widetilde{Q}_{\mathrm{f}} \\
\widetilde{Q}_{\mathrm{s}}
\end{array}\right), \quad\left(\begin{array}{c}
\widetilde{Q}_{\mathrm{f}} \\
\widetilde{Q}_{\mathrm{s}}
\end{array}\right)\left(\begin{array}{ll}
Q_{\mathrm{f}} & Q_{\mathrm{s}}
\end{array}\right)=\left(\begin{array}{cc}
I & 0 \\
0 & I
\end{array}\right),
$$

where matrices $Q_{\mathrm{f}}$ and $Q_{\mathrm{s}}$ correspond to the fast and slow subspaces ( $Q_{\mathrm{f}}$ is a matrix $n \times n_{\mathrm{f}}$ of the fast eigenvectors, $Q_{\mathrm{s}}$ is a matrix $n \times n_{\mathrm{s}}$ of the slow eigenvectors, $\widetilde{Q}_{\mathrm{f}}$ is a matrix $n_{\mathrm{f}} \times n$ and $\widetilde{Q}_{\mathrm{s}}$ is a matrix $\left.n_{\mathrm{S}} \times n\right)$. The parameter $\tau$ is a time-scale splitting parameter. This splitting parameter determines the dimensions of the slow $\left(n_{\mathrm{S}}\right)$ and fast $\left(n_{\mathrm{f}}=n-n_{\mathrm{S}}\right)$ subspaces.

Using a standard linearization of the RHS of (2.3.1) at the point $\mathrm{Z}_{0}$, we get

$$
\frac{\mathrm{d} Z}{\mathrm{~d} t}=\Phi(Z) \approx \Phi\left(Z_{0}\right)+\left.\frac{\partial \Phi}{\partial Z}\right|_{Z=Z_{0}}\left(Z-Z_{0}\right) .
$$


The Jacobian at the point $Z_{0}$ can be represented as a product of three matrices: the transition matrix $Q$, a two-block representation $J_{M}$ of the Jacobian $\left(M_{\mathrm{J}}\right)$ in the eigenvectors basis and inverse of the transition matrix $Q^{-1}$

$$
\left.\frac{\partial \Phi}{\partial Z}\right|_{Z=Z_{0}}=M_{\mathrm{J}}\left(Z_{0}\right)=Q J_{M_{\mathrm{J}}} Q^{-1}=M_{\mathrm{J}}=\left(\begin{array}{ll}
Q_{\mathrm{f}} & Q_{\mathrm{s}}
\end{array}\right)\left(\begin{array}{cc}
J_{M_{\mathrm{f}}} & 0 \\
0 & J_{M_{\mathrm{s}}}
\end{array}\right)\left(\begin{array}{l}
\widetilde{Q}_{\mathrm{f}} \\
\widetilde{Q}_{\mathrm{s}}
\end{array}\right) .
$$

The square matrix $J_{M_{\mathrm{J}}}$ is decomposed into two-block structured matrices that correspond to fast and slow invariant subspaces. The matrix $J_{M_{\mathrm{J}}}$ is $n \times n, J_{M_{\mathrm{s}}}$ is $n_{\mathrm{s}} \times n_{\mathrm{s}}$ and $J_{M_{\mathrm{f}}}$ is $n_{\mathrm{f}} \times n_{\mathrm{f}}$.

Introduce the intermediate variable $\phi=Z-Z_{0}$ and re-write the previous expression in the form

$$
\frac{\mathrm{d} \phi}{\mathrm{d} t}=\Phi\left(Z_{0}\right)+M_{\mathrm{J}}\left(Z_{0}\right) \phi=\Phi\left(Z_{0}\right)+Q\left(Z_{0}\right) J_{M_{\mathrm{J}}}\left(Z_{0}\right) Q^{-1}\left(Z_{0}\right) \phi .
$$

Multiply both sides of the equation by the inverse matrix $Q^{-1}\left(Z_{0}\right)$

$$
Q^{-1}\left(Z_{0}\right) \frac{\mathrm{d} \phi}{\mathrm{d} t}=Q^{-1}\left(Z_{0}\right) \Phi\left(Z_{0}\right)+J_{M_{\mathrm{J}}}\left(Z_{0}\right) Q^{-1}\left(Z_{0}\right) \phi
$$

and introduce the new variable (this is a point of the transition from the original variables to the new ones, which allow to decompose fast and slow motions—-see (2.3.2) in the previous section)

$$
\Psi=Q^{-1}\left(Z_{0}\right) \phi
$$

With respect to the new variable, the equation can be written in the form

$$
\frac{\mathrm{d} \Psi}{\mathrm{d} t}=\phi \frac{\mathrm{d}\left(Q^{-1}\left(Z_{0}\right)\right)}{\mathrm{d} t}+Q^{-1}\left(Z_{0}\right) \Phi\left(Z_{0}\right)+J_{M_{\mathrm{J}}}\left(Z_{0}\right) \Psi .
$$

One can show that the first term in the RHS of (2.4.8) is negligible under certain special conditions (Maas, 1994; Maas \& Pope, 1992) (compare with the condition (2.3.8)). Equation (2.4.8) is reduced to the simple equation in the form (recalling that the Jordan form $J_{M_{\mathrm{J}}}$ of the Jacobian $\left(M_{\mathrm{J}}\right)$ is decomposed into two invariant subspaces)

$$
\frac{\mathrm{d} \Psi}{\mathrm{d} t}=Q^{-1}\left(Z_{0}\right) \Phi\left(Z_{0}\right)+J_{M_{\mathrm{J}}}\left(Z_{0}\right) \Psi
$$

If

$$
\widetilde{Q}_{\mathrm{f}}\left(Z_{0}\right) \Phi\left(Z_{0}\right)=0
$$

then with the initial condition $\Psi_{\mathrm{f}}=0$, when $\Psi=\left(\psi_{\mathrm{f}}, \psi_{\mathrm{s}}\right)^{\top}$, the fast variables will remain zero for $t>0$. Thus, according to the original algorithm of Maas and Pope (1992), the ILDM is determined by an underdetermined system of $n_{\mathrm{f}}$ equations for $n$ unknowns

$$
\widetilde{Q}_{\mathrm{f}}(Z) \Phi(Z)=0
$$

which corresponds to the relation (2.3.10) of the general framework.

To recap the description of the ILDM approach, it would be worthwhile to interpret the aforementioned procedure into the geometric language. Indeed, the ILDM approach represents a numerical algorithm of creation of a new global coordinate system, in which the treated system of ODEs is transformed to the conventional form of the SPS. The creation of the new coordinate system is based on the local determination of the fast and slow directions (in the new desired metrics, these directions should serve as coordinates). Application of ILDM algorithm contains the following essential steps: (a) a determination of a dimension of a fast vector subfield (equal to a number of 'big' eigenvalues of Jacobi matrix); (b) introduction of transition matrix (transition to invariant fast and slow subspaces) and (c) analysis of restrictions (for practical situations, a variation of transition matrix is small enough). 


\section{Problematic points-application of ILDM}

In this section, we will consider an application of ILDM technique to some simple examples, which were chosen to illustrate the restrictions of the existing version of the ILDM (as we will see, further ILDM approach does not properly work on the examples). Our aim here is to realize the reasons of these unexpected results of the ILDM application.

Let us start from the general discussion. The condition (2.3.8) imposes definite restrictions on the type of the system of ODEs, which can be treated by the approach, presented in Section 2.3. In particular, one can deduce that the linear systems of ODEs are the most suitable object of a possible application of the ILDM technique. Indeed, a constant vector field $\Phi(Z)$ provides us with the constant eigenvectors and the constant transition matrix $\widetilde{Q}$. This automatically satisfies the condition (2.3.8)-a derivative of the matrix $\widetilde{Q}$ with respect to time $t$ equals to zero. Therefore, it would be natural to expect that the ILDM procedure would work properly being applied to linear systems of ODEs. Two further sections show that our expectations were groundless.

\subsection{Linear planar system-real eigenvalues}

The next example demonstrates that even for SPSs, division into fast and slow eigenvectors not necessary exists. Let us start from a simplest possible non-trivial case of the system (2.3.1)

$$
\left\{\begin{array}{l}
\dot{x}(t)=x+\frac{1}{\varepsilon} y, \quad \frac{\mathrm{d} Z}{\mathrm{~d} t}=\Phi(Z), \quad Z=\left(\begin{array}{l}
x \\
y
\end{array}\right), \quad \Phi(Z)=A Z, \quad A=\left(\begin{array}{ll}
1 & \frac{1}{\varepsilon} \\
0 & 1
\end{array}\right), \quad \varepsilon \ll 1 .
\end{array}\right.
$$

It is obvious that the Jacobian in the considered system equals to the matrix $A$. Eigenvalues of the matrix $A$ are real and they are equal to each other (unity). According to the traditional interpretation of the ILDM approach, this fact witnesses that there is no internal hierarchy in the considered system (3.1.1) and the analysis cannot be further proceeded. Nevertheless, as one can readily see, the original system (3.1.1) has essentially different rates of change (at least in the region, where functions $x(t)$ and $y(t)$ are of the same order: $x(t)$ is fast and $y(t)$ is slow). Moreover, multiplying by $\varepsilon$ the first equation of the system (3.1.1), we can re-write it in the conventional SPS form.

$$
\Phi(Z)=\left(\begin{array}{c}
x+\frac{1}{\varepsilon} y \\
y
\end{array}\right)=\left(\begin{array}{l}
\Phi_{\mathrm{f}}(Z) \\
\Phi_{\mathrm{s}}(Z)
\end{array}\right), \quad \Phi_{\mathrm{f}}(Z)=x+\frac{1}{\varepsilon} y, \quad \Phi_{\mathrm{s}}(Z)=y .
$$

This example leads us to the conclusion that even in the simplest linear cases with strongly determined hierarchy, the eigenvalues of the Jacobian do not always provide us with correct information regarding possible reduction to singularly perturbed form.

\subsection{Linear planar system-complex eigenvalues}

The main idea of this 2D example is to demonstrate a drawback of the ILDM approach in the case of an existence of complex eigenvalues of a corresponding Jacobi matrix. Consider the following planar version of the system (2.3.1)

$$
\left\{\begin{array}{l}
\dot{x}(t)=y, \\
\dot{y}(t)=-\frac{1}{\varepsilon} x+y,
\end{array} \quad \Phi(Z)=A Z, \quad Z=\left(\begin{array}{l}
x \\
y
\end{array}\right), \quad A=\left(\begin{array}{ll}
0 & 1 \\
-\frac{1}{\varepsilon} & 1
\end{array}\right), \quad \varepsilon \ll 1 .\right.
$$


Similar to the example studied in Section 3.1, the system (3.2.1) can be easily written in the conventional form of the SPS. Nevertheless, the ILDM method cannot produce any ILDM because the real parts of the two complex eigenvalues are equal

$$
\lambda^{2}-\lambda+\frac{1}{\varepsilon}=0, \quad \lambda_{1,2}=\frac{1}{2}\left(1 \pm \sqrt{1-\frac{4}{\varepsilon}}\right)=\frac{1}{2}\left(1 \pm \mathrm{i} \sqrt{\frac{4}{\varepsilon}-1}\right), \quad \frac{4}{\varepsilon}-1>0
$$

and, therefore, there is no distinction in rates of change of the two processes involved.

In the geometrical language, the traditional ILDM procedure fails to decompose the vector field $\Phi(Z)$ (determined by the RHS of the system (3.2.1)) into two components: fast and slow. This unexpected result looks a bit confusing, especially in the light of the evident decomposition of the original vector field into the fast and slow components

$$
\Phi(Z)=\left(\begin{array}{c}
y \\
-\frac{1}{\varepsilon} x+y
\end{array}\right)=\left(\begin{array}{l}
\Phi_{\mathrm{s}}(Z) \\
\Phi_{\mathrm{f}}(Z)
\end{array}\right)
$$

\subsection{Summary}

To summarize the analysed above two linear examples and other studies (Goldfarb et al., 2004), we can formulate shortly two main practical problems of the ILDM technique, which are illustrated by the following examples:

1. In the case of complex eigenvalues (or when the real parts of the eigenvalues are close), directions of 'fast' and 'slow' motions obtained by the ILDM algorithm can be very different from 'fastslow' motions of the original system.

2. Transition zones (turning manifolds) between different invariant manifolds cannot be correctly described by ILDM methods because for these zones division onto 'big' and 'small' eigenvalues typically do not exist.

On the other hand, it was proved (Rhodes et al., 1999) that in a small neighbourhood of a stable invariant manifold for an SPS, there exists an ILDM. Moreover, numerical simulation demonstrates that in small neighbourhoods of invariant manifolds, this conclusion is correct.

\section{TILDM-suggested modification of ILDM}

Suppose for a moment that we are asked to investigate a dynamical behaviour of some arbitrary system of ODEs, which is NOT written in the conventional form (2.1.1)-(2.1.2) of the SPS (say, it has a general form (2.3.1)). Common sense states that there are only two possibilities: (i) the system under consideration does not have internal hierarchy and (ii) the considered system does represent a multi-scale system. Let us focus on the second option as on the most interesting one from the point of view of an asymptotic analysis. An existence of a dispersion of time-scales of the ODEs of the system allows us to suggest that there is a system of coordinate (differing from the original one), where the given system of ODEs can be re-written in the conventional SPS form (2.1.1)-(2.1.2). Ideally, this desired system of new coordinates should have a number $\left(n_{\mathrm{f}}\right)$ of axes, directions which are close to the directions of the slow motion, and direction of the others $\left(n_{\mathrm{S}}\right)$ —close to the fast motions. Our task may be formulated as to find this system of new coordinates and to determine a transformation transforming the original variables into the new ones. ILDM method is supposed to give an answer to this question. Recalling that it uses matrix built from the eigenvectors of the Jacobian of the RHS of the original system as a transformation matrix (see Section 2.4 for details). 


\subsection{Main idea of TILDM}

Let us explain shortly a main idea of the suggested modification of the ILDM method (which can be called as TILDM) on the simple 2D case (we will see further what the sense of the letter T is).

It is clear that the Jacobian of the original system contains a full information concerning the system dynamics in the case of the linear vector field $\Phi$ (2.3.1) only. Assuming that $\Phi$ is a linear mapping, we can conclude that it has directions of the maximal and minimal stretch, which in the accepted language of the present paper can be interpreted as the fast and slow directions. Therefore, it would be ideal, if we could build a transformation, which converts the original coordinates (variables) into these fast and slow directions. The latter can be found using a general theory of linear algebra. It teaches that an arbitrary linear non-degenerated transformation (mapping) maps the unit circle with the centre in the origin to an ellipse with the centre in the origin. Hence, we can identify the directions of the fast and slow motions with directions of the large and small semi-axes of the ellipse, respectively. To build the desired transformation, remember that elementary linear algebra provides us with a well-known technical procedure of a determination of the semi-axes of the ellipse. Consider a matrix $M$ of an arbitrary non-degenerate linear transformation and its transpose matrix $M^{*}$. The eigenvalues of the matrix $M M^{*}$ represent the length squares of the ellipse's semi-axes and the corresponding eigenvectors coincide with the directions of the semi-axes. One of the distinguishing features of the matrix of the type $M M^{*}$ is an absence of complex eigenvalues (all eigenvalues are real and corresponding eigenvectors are orthogonal). Assuming that the Jacobian $J$ of the considered system contains the relevant information regarding the system dynamics (hierarchy of the system), we can conclude that the matrix of the transformation chosen as $J J^{*}$ provides us with more accurate information regarding the subdivision that the Jacobian $J$ does.

\subsection{Description of the TILDM algorithm}

Note here that the main purpose of the suggested TILDM method coincides with that of the ILDM approach: our aim is a determination of the 'fast' and 'slow' directions (subspaces, subsystems) and a construction of the corresponding TILDM for the system (2.3.1).

Consider the system (2.3.1) and let us describe here very briefly the essential analytical steps of the TILDM construction following the same scheme as for the ILDM case (see Section 2.4). For an arbitrary point $Z$ :

1. Build matrix $M(Z)=J(Z) J^{*}(Z)$ and determine its eigenvalues $\lambda_{i}(i=1,2, \ldots, n)$.

2. Check whether a splitting exists (its absence means that at that point the suggested procedure is inapplicable). If 'yes', re-order the obtained eigenvalues as follows

$\lambda_{i} \gg \tau>\lambda_{j} ; \tau>0, i=1, \ldots, n_{\mathrm{f}}, j=n_{\mathrm{f}}+1, \ldots, n$.

3. Build matrices $Q(Z)$ and $Q^{-1}(Z)=\widetilde{Q}(Z)$ along the rules used during a derivation of (2.4.2).

4. Obtain a system of equations $\widetilde{Q}_{\mathrm{f}}(Z) \Phi(Z)=0$ describing the TILDM.

The aforementioned formalized algorithm needs some comments. Due to the stated above property of the symmetric matrix, the transformation matrix $M(Z)=J(Z) J^{*}(Z)$ has no complex eigenvalues. It means that we are able to establish a relation between the processes involved in the whole phase space (and not in the region only, where the eigenvalues of the Jacobian are real, as in the conventional ILDM procedure). On the other hand, this evident advantage has its own drawback-all eigenvalues are positive, i.e. an information regarding the stability of the corresponding parts of the unknown manifolds is lost. Nevertheless, an analysis of the stability can be performed on the basis of the suitable analysis of the Jacobi matrix $J$ at any point $Z_{0}$ that belongs to TILDM. Once a spectrum of $\widetilde{Q}_{\mathrm{f}}\left(Z_{0}\right) J Q_{\mathrm{f}}\left(Z_{0}\right)$ 
has negative real parts, we can deduce that the corresponding TILDM is stable. In any other case, the corresponding TILDM is unstable. Remarks regarding necessary smallness of the variations of the matrix field $Q$ (Section 2.3) still remains crucial for TILDM as well as for ILDM.

\subsection{Asymptotics-planar case}

A general theorem can be formulated, which states that the aforementioned TILDM algorithm results in the surface, which asymptotically tends to the slow manifold. The rigorous proof of the general theorem regarding an arbitrary system of ODEs with internal hierarchy represents a rather complicated problem and cannot be performed in the framework of the present work. Nevertheless, it is possible to demonstrate the essence of the suggested method analysing the simple planar version of the general system. This will allow the authors to present the main idea of the proof, whereas the rigorous mathematical description will be the subject of future publications.

Consider the SPS of the type (2.3.1) in the planar case. It reads

$$
\frac{\mathrm{d} z}{\mathrm{~d} t}=\Phi(x, y), \quad z=\left(\begin{array}{c}
x(t) \\
y(t)
\end{array}\right), \quad \Phi(x, y)=\left(\begin{array}{c}
\frac{1}{\varepsilon} f(x, y) \\
g(x, y)
\end{array}\right) .
$$

Following the algorithm presented in Section 4.2, we build Jacobian $J$, its conjugate $J^{*}$ and their multiplication (transformation matrix $M$ )

$$
\begin{gathered}
J(x, y)=\left(\begin{array}{cc}
\frac{f_{x}}{\varepsilon} & \frac{f_{y}}{\varepsilon} \\
g_{x} & g_{y}
\end{array}\right), \quad J^{*}(x, y)=\left(\begin{array}{cc}
\frac{f_{x}}{\varepsilon} & g_{x} \\
\frac{f_{y}}{\varepsilon} & g_{y}
\end{array}\right), \\
M=J(x, y) J^{*}(x, y)=\left(\begin{array}{ll}
M_{11} & M_{12} \\
M_{21} & M_{22}
\end{array}\right)=\left(\begin{array}{cc}
\frac{f_{x}^{2}+f_{y}^{2}}{\varepsilon^{2}} & \frac{f_{x} g_{x}+f_{y} g_{y}}{\varepsilon} \\
\frac{f_{x} g_{x}+f_{y} g_{y}}{\varepsilon} & g_{x}^{2}+g_{y}^{2}
\end{array}\right) .
\end{gathered}
$$

Eigenvectors (non-normalized) of the transformation matrix $M$, the matrix $Q$ and its inverse $Q^{-1}$ have the following form

$$
\begin{gathered}
\lambda_{1,2}=\frac{1}{2}\left(\operatorname{Tr}(M) \mp \sqrt{(\operatorname{Tr}(M))^{2}-4 \operatorname{Det}(M)}\right), \\
\operatorname{Tr}(M)=M_{11}+M_{22}, \quad \operatorname{Det}(J)=M_{11} M_{22}-M_{12} M_{21}, \\
\lambda=\lambda_{1} \Rightarrow \zeta_{1}=\left(\begin{array}{c}
-M_{12} \\
M_{11}-\lambda_{1}
\end{array}\right), \quad \lambda=\lambda_{2} \Rightarrow \zeta_{2}=\left(\begin{array}{c}
-M_{12} \\
M_{11}-\lambda_{2}
\end{array}\right) .
\end{gathered}
$$

To determine, which of the two eigenvalues is the largest, it is worthwhile to expand the expressions with respect to the small parameter of the problem $(\varepsilon)$. The expansions look as follows

$$
\begin{aligned}
& \lambda_{\mathrm{f}}=\frac{f_{x}^{2}+f_{y}^{2}}{\varepsilon^{2}}+\frac{\left(f_{x} g_{x}+f_{y} g_{y}\right)^{2}}{f_{x}^{2}+f_{y}^{2}}+\mathrm{O}\left(\varepsilon^{2}\right), \\
& \lambda_{\mathrm{s}}=\frac{\left(f_{y} g_{x}-f_{x} g_{y}\right)^{2}}{f_{x}^{2}+f_{y}^{2}}+\mathrm{O}\left(\varepsilon^{2}\right) .
\end{aligned}
$$

At the present stage of the analysis, we can build matrices $Q$ and $Q^{-1}$. They read

$$
Q=\left(\begin{array}{cc}
-M_{12} & -M_{12} \\
M_{11}-\lambda_{1} & M_{11}-\lambda_{2}
\end{array}\right), \quad Q^{-1}=\frac{1}{\operatorname{Det}[Q]}\left(\begin{array}{rr}
-M_{11}-\lambda_{2} & -M_{12} \\
M_{11}-\lambda_{1} & -M_{12}
\end{array}\right) .
$$


The TILDM, in accordance to the aforementioned algorithm, is given by the equation $\widetilde{Q}_{\mathrm{f}} \Phi$ (recalling here that the matrix $Q^{-1}$ is represented in the form (2.4.2)). To realize the mathematical sense of the cumbersome derived relations, let us expand the two expressions $\widetilde{Q}_{\mathrm{s}} \Phi$ and $\widetilde{Q}_{\mathrm{f}} \Phi$ with respect to the small parameter $\varepsilon$. The expansions can be written in the form

$$
\begin{aligned}
\widetilde{Q}_{\mathrm{s}} \Phi(Z)= & \frac{\left(f_{x}^{2}+f_{y}^{2}\right) g-f_{x} g_{x} f-f_{y} g_{y} f}{f_{x}^{2}+f_{y}^{2}}+\mathrm{O}\left(\varepsilon^{2}\right), \\
& \widetilde{Q}_{\mathrm{f}} \Phi(Z)=\frac{\left(f_{x}^{2}+f_{y}^{2}\right) f}{\left(f_{x} g_{x}+f_{y} g_{y}\right) \varepsilon^{2}} \\
& g-\frac{f\left(f_{y} g_{x}-f_{x} g_{y}\right)^{2}}{\left(f_{x}+f_{y}\right)^{2}\left(f_{x} g_{x}+f_{y} g_{y}\right)}
\end{aligned}
$$

As one can easily see, the term of leading order in the expressions (4.3.7) for $\widetilde{Q}_{\mathrm{f}} \Phi$ is proportional to $\varepsilon^{-2}$. In addition, its nominator contains two multipliers, one of them-the function $f$

$$
\widetilde{Q}_{\mathrm{f}} \Phi(Z) \approx \frac{\left(f_{x}^{2}+f_{y}^{2}\right) f}{\left(f_{x} g_{x}+f_{y} g_{y}\right)}
$$

Equating the RHS of (4.3.8) to zero gives us two options: (i) $f=0$ and (ii) a nominator of the ratio in the RHS of (4.3.8) is equal to zero. The first one of these two options naturally provides us with the conclusion that in the zeroth approximation, the TILDM coincides with the slow curve of the system (2.3.1) in the planar case.

Note here that the second option has its own sense, but its detailed analysis lies beyond the scope of the present piece of work. Let us remark here that in fact the single equation $f_{x}^{2}+f_{y}^{2}=0$ is equivalent to a system of the two new separate equations: $f_{x}=0$ and $f_{y}=0$. In the geometrical language, an existence of the two independent equations means that we have possibly problematic points (instead of curves in the case of the single equation in a plane).

This outcome justifies our suggestion to use a multiplication of the Jacobi matrix and its conjugate as a transformation matrix for transformation to a new coordinate system, where there is distinction between rates of change of different directions.

\section{TILDM-how it works}

In this section, we will demonstrate how the suggested TILDM method works in a number of particular cases, where the traditional ILDM approach faces problems.

\subsection{Linear example-Jacobian with real eigenvalues}

Consider the linear example (3.1.1) (Section 3.1) and apply the suggested TILDM approach. The Jacobi matrix $J$, its conjugate $J^{*}$ and the transformation matrix $M$ read

$$
J(x, y) \stackrel{\text { by def }}{=} A=\left(\begin{array}{ll}
1 & \frac{1}{\varepsilon} \\
0 & 1
\end{array}\right), \quad J^{*}(x, y) \stackrel{\text { by def }}{=} A^{*}=\left(\begin{array}{cc}
1 & 0 \\
\frac{1}{\varepsilon} & 1
\end{array}\right), \quad J J^{*}=\left(\begin{array}{cc}
1+\frac{1}{\varepsilon^{2}} & \frac{1}{\varepsilon} \\
\frac{1}{\varepsilon} & 1
\end{array}\right) .
$$


The eigenvalues of the transformation matrix $M$ are both real (please note that the matrix $M$ is symmetric according to its definition) and look as follows

$$
\lambda_{1,2}=\frac{1}{2}\left(\left(2+\frac{1}{\varepsilon^{2}}\right) \mp \sqrt{\left(2+\frac{1}{\varepsilon^{2}}\right)^{2}+\frac{4}{\varepsilon^{2}}}\right) .
$$

The expansions of the eigenvalues with respect to the small parameter of the system read (the leading orders of asymptotics are presented)

$$
\begin{gathered}
\lambda_{1}=\frac{1}{\varepsilon^{2}}+2-\varepsilon^{2}+\mathrm{O}\left(\varepsilon^{3}\right), \quad \lambda_{2}=\varepsilon^{2}+\mathrm{O}\left(\varepsilon^{3}\right) \\
e_{1}=\left(\begin{array}{l}
1 \\
\varepsilon
\end{array}\right), \quad e_{2}=\left(\begin{array}{r}
-\varepsilon \\
1
\end{array}\right) .
\end{gathered}
$$

It can be readily seen that the eigenvectors (5.1.4) coincide with the corresponding directions of the fast and slow motions: the eigenvector $e_{1}$ is asymptotically close to the direction of the fast motion (parallel to the $x$-axis), whereas the eigenvector $e_{2}$ is orthogonal to $e_{1}$.

The equation for TILDM reads

$$
\Phi_{\mathrm{f}}^{*}(Z)=x+\frac{1}{\varepsilon} y+\varepsilon y=0 .
$$

Note that (5.1.5) differs from (3.1.2) (the term $\varepsilon y$ is added) and coincides with the slow curve equation (see Section 3.1) up to the first order of magnitude by $\varepsilon$.

\subsection{Linear example-Jacobian with complex eigenvalues}

The situation with the second linear example (Section 3.2) appears to be a bit simpler. A corresponding symmetric matrix $M$ (transformation matrix) reads

$$
J(x, y) \stackrel{\text { by def }}{=} A=\left(\begin{array}{rr}
0 & 1 \\
-\frac{1}{\varepsilon} & 1
\end{array}\right), \quad J^{*}(x, y) \stackrel{\text { by def }}{=} A^{*}=\left(\begin{array}{cc}
0 & -\frac{1}{\varepsilon} \\
1 & 1
\end{array}\right), \quad M=J J^{*}=\left(\begin{array}{cc}
1 & 1 \\
1 & 1+\frac{1}{\varepsilon^{2}}
\end{array}\right) .
$$

The eigenvalues are real and equal to (for the leading order of magnitude)

$$
\begin{gathered}
\lambda_{1,2}=\frac{1}{2}\left(\left(2+\frac{1}{\varepsilon^{2}}\right) \mp \sqrt{\left(2+\frac{1}{\varepsilon^{2}}\right)^{2}+4}\right), \\
\lambda_{1}=\frac{1}{\varepsilon^{2}}+1+\varepsilon^{2}+\mathrm{O}\left(\varepsilon^{3}\right), \quad \lambda_{2}=1-\varepsilon^{2}+\mathrm{O}\left(\varepsilon^{3}\right),
\end{gathered}
$$

and the corresponding eigenvectors

$$
e_{1}=\left(\begin{array}{c}
\varepsilon^{2} \\
1
\end{array}\right), \quad e_{2}=\left(\begin{array}{c}
1 \\
-\varepsilon^{2}
\end{array}\right)
$$

It is not hard to see that in this example as well as in previous examples, fast and slow directions are close to original ones; moreover, the last example shows that the problem of complex eigenvalues does not exist for the modification of the ILDM. 
The equation for TILDM reads

$$
\Phi_{\mathrm{f}}^{*}(Z)=-\frac{1}{\varepsilon} x+y+\varepsilon^{2} y=0
$$

Note that similar to the example studied in Section 5.1, (5.2.5) differs from (3.2.3) (the term $\varepsilon^{2} y$ is added) and coincides with the slow curve equation (see Section 3.2) up to the second order of magnitude by $\varepsilon$.

\subsection{Artificial example}

This section illustrates a well-known unwritten regularity stating that a good theory is normally valid far beyond formal bounds of its applicability. As it was already pointed out, the application of the suggested TILDM approach is formally restricted by the linear differential systems. Nevertheless, it turns out that the proposed technique succeeds to reach the desired aims in much more complex problems.

Consider a system of the type (2.3.1), where the vector field $\Phi(Z)$ reads

$$
\Phi(Z)=\left(\begin{array}{c}
-\frac{1}{\varepsilon}(x+\sin (x)+\sin (y)) \\
-y
\end{array}\right), \quad \varepsilon \ll 1 .
$$

The Jacobian of the vector field (5.3.1) looks as follows

$$
J=\left(\begin{array}{cc}
\frac{-1-\cos (x)}{\varepsilon} & \frac{-\cos (y)}{\varepsilon} \\
0 & -1
\end{array}\right)
$$

and due to its upper triangular structure its eigenvalues are evident

$$
\lambda_{1}=\frac{-1-\cos (x)}{\varepsilon}, \quad \lambda_{2}=-1 .
$$

At a first glance, it seems that a relation between these two eigenvalues is obvious: $\lambda_{1}$ is the larger eigenvalue and $\lambda_{2}$ is the smaller one. A bit more deeper consideration shows that there are infinite number of regions (vertical bands close to the points $\pi+2 k \pi)$ in the $(x, y)$ plane, where values of the expression $(\cos (x)+1)$ are of the order of $\varepsilon$ and smaller. This means that according to ILDM (!), the system's hierarchy (which is valid in most points of the plane) changes to the opposite in these regions. This misleading outcome causes the fact that ILDM provides us with a number of artificial manifolds (which can be conditionally called 'ghosts') and a wrong fast direction. Figure 1(a) demonstrates an existence of the artificial manifolds (dotted curves), obtained as a result of ILDM run on the system (5.3.1).

In the present example, there are no complex-valued eigenvalues. Nevertheless, there are domains where a direction of the real fast motion is almost perpendicular to that produced by the ILDM approach and this is the reason for appearance of the 'ghosts' - artificial manifolds, which have no sense in the system dynamics. A discrimination of these 'artificial' objects represents one of the main complications for a regular use of the ILDM method.

The real slow manifold of the system under consideration is located in the vicinity of the $y$-axis (one of the dotted curves, produced by the ILDM, lies close to it). The dashed curve represents the slow curve (zeroth approximation of the real slow manifold, Fig. 1(b)), which can be derived according to the MIM approach and the equation of which reads

$$
x+\sin (x)+\sin (y)=0 .
$$


(a)

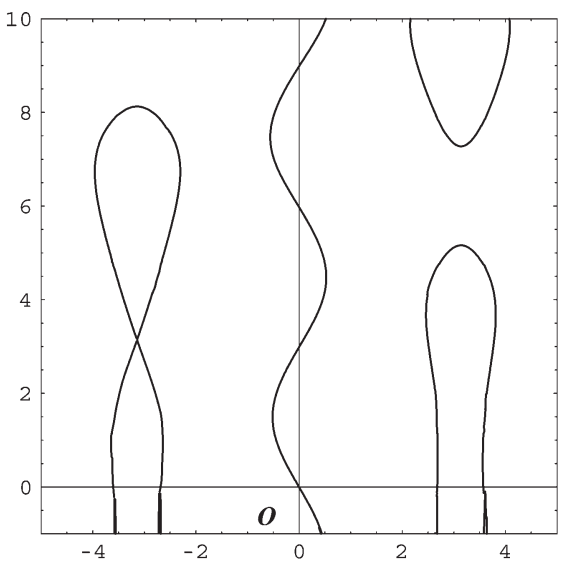

(b)

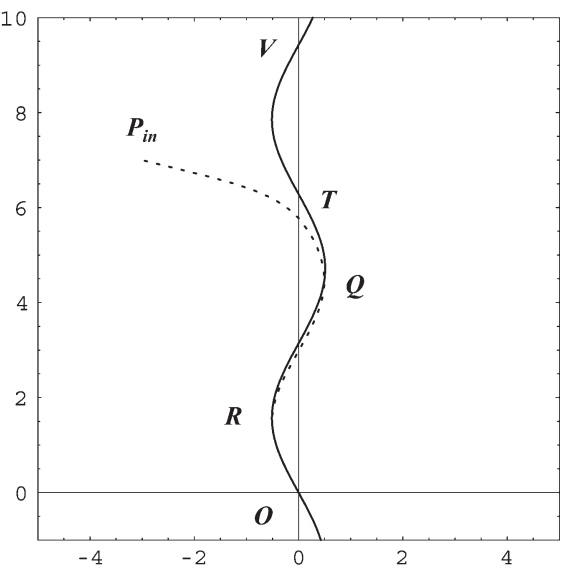

(c)

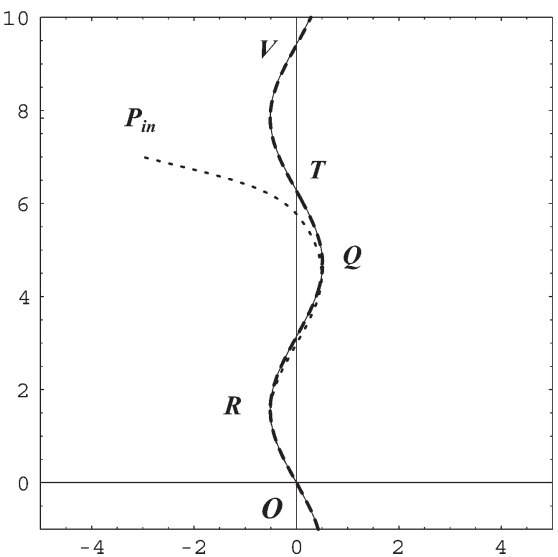

FIG. 1. Characteristic curves and a typical trajectory of the system (5.3.1) in the state plane $(x, y)$. Letters denote: (a) results of application of the TILDM procedure, approximation of the real manifold (curve, close to the axis $y$ ) and ghost objects (dotted curves); (b) slow curve (solid curve) and part of the results of TILDM running (dashed curve); (c) solid curve and the results of TILDM running (solid and dashed coinciding curves, respectively), a typical trajectory starting from the point $P_{\text {in }}$. 
Figure 1(b) depicts the slow curve (solid line) of the system, the part of ILDM running results close to $y$-axis (dashed) and the trajectory of the system (dotted), which starts at some chosen point in the $(x, y)$ plane. One can easily see that a trajectory is naturally subdivided into the two different stages. Within the first one, the trajectory moves fast from the initial point $P_{\text {in }}$ towards the slow manifold. Once the system trajectory approached the close proximity of the slow manifold, it begins to move along the manifold being 'glued' to it.

Figure 1(c) compares the slow curve (solid) and the results of the TILDM running (dashed). We can readily see that the two curves coincide with a rather good accuracy. Moreover, TILDM does not produce any artificial objects like ILDM does. This justifies our previous theoretical conclusion regarding better ability of the TILDM approach proposed here to determine the slow manifolds of the multi-scale systems.

\subsection{Semenov's model of thermal explosion}

In this section, we consider a realistic example taken from the mathematical theory of combustionSemenov's model of thermal explosion with heat losses.

In the dimensionless form, the relevant mathematical model of this classical problem (Semenov, 1928, Frank-Kamenetskii, 1969) can be presented as

$$
\begin{gathered}
\gamma \frac{\mathrm{d} \theta}{\mathrm{d} t}=\eta \exp \left(\frac{\theta}{1+\beta \theta}\right)-\delta \theta=F_{1}(\theta, \eta), \quad \theta(0)=0 \\
\frac{\mathrm{d} \eta}{\mathrm{d} t}=-\eta \exp \left(\frac{\theta}{1+\beta \theta}\right), \quad \eta(0)=1 .
\end{gathered}
$$

Here, $\theta$ is a dimensionless temperature, $\eta$ is a dimensionless concentration, $\delta$ is a dimensionless heat loss parameter, $\gamma$ is a reciprocal of the dimensionless adiabatic temperature rise and $\beta$ is a dimensionless ambient temperature. The parameters $\gamma$ and $\beta$ are normally small with respect to unity and $\gamma$ serves as the singular parameter of the system. Therefore, the system (5.4.1)-(5.4.2) can be treated as a multi-scale system.

Note that the dynamics of the system (5.4.1)-(5.4.2) is known well from previous studies. In particular, the detailed analysis of the considered Semenov model was performed in the previous paper of the authors (Goldfarb et al., 2004). The scrutiny of the system dynamics along the lines of the MIM approach and detailed analysis of the application of the ILDM technique are presented there. Therefore, here we restrict ourselves by the short summary of the results only.

It is worthwhile here to determine the slow curve of the system (5.4.1)-(5.4.2) before an application of TILDM. According to Gol'dshtein \& Sobolev $(1992,1988)$, the slow curve $\Omega_{1}$ of the system (5.4.1)(5.4.2) is given by

$$
F_{1}(\eta, \theta) \equiv \eta \exp \left(\frac{\theta}{1+\beta \theta}\right)-\delta \theta=0 .
$$

Let us now compare the results of the applications of the suggested TILDM procedure and ILDM technique. Figure 2(a) shows that the TILDM (thick dashed line) coincides almost exactly with the slow curve $\Omega_{1}$ (thin solid line). A good accuracy of the approximation of the slow curve $\Omega_{1}$ by the TILDM witnesses in favour of the suggested algorithm. The thin dotted line represents the trajectory of the system (numerical simulation). It starts at the point $I(0,1)$ and moves fast towards the attractive branch of the manifold with the slow variable remaining practically constant (fast stage $I N, \eta=1$ ). Far from the 


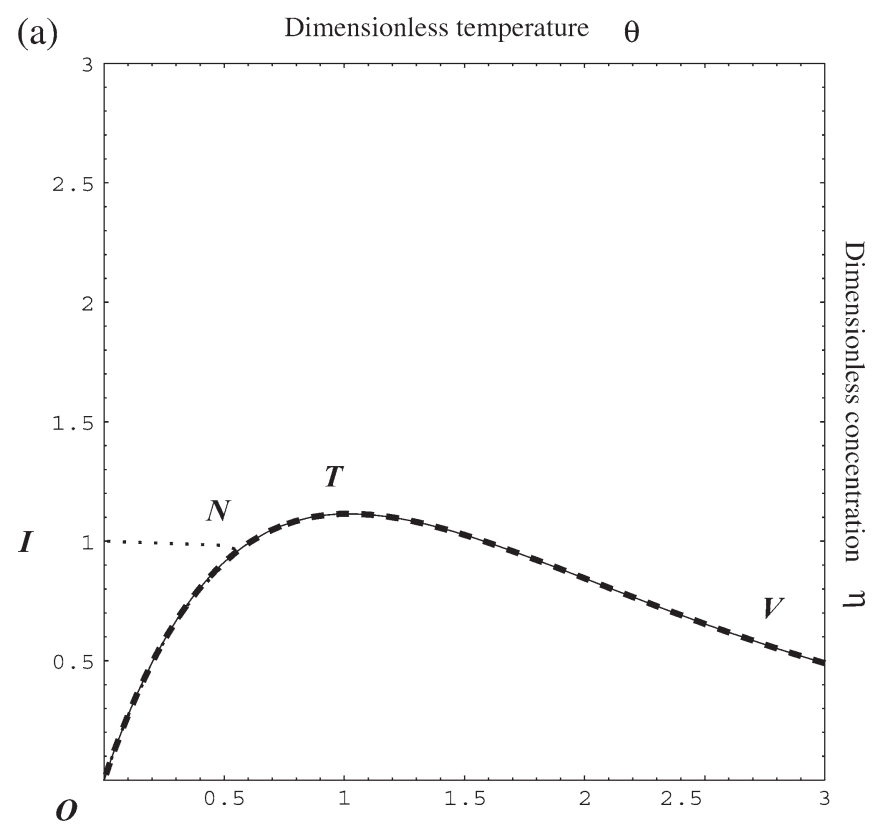

(b) Dimensionless temperature $\theta$

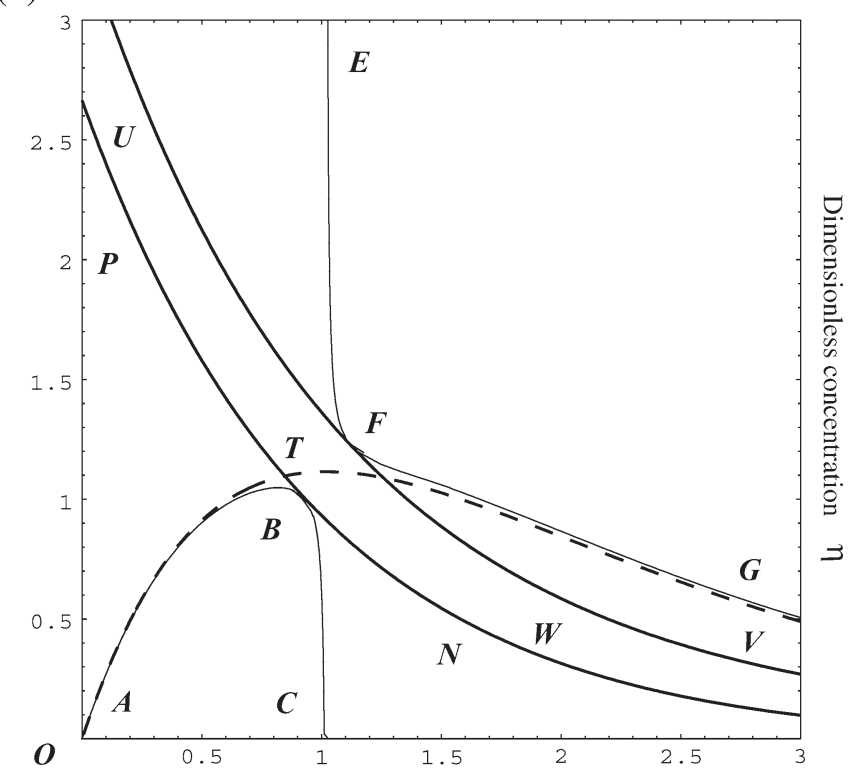

FIG. 2. The characteristic curves and the typical trajectories of the system (5.4.1)-(5.4.2) in the state plane $\theta-\eta$. (a) Slow curve (thin solid curve), TILDM (bold dashed line) and sample numerical trajectory (dotted line); (b) slow curve (dashed line), results of application of the TILDM procedure, region of the complex eigenvalues (band bounded by $P N$ and $U W$ ). 
slow manifold, the trajectory is characterized by the constant (asymptotically) value of the slow variable ( $\eta=1$ in the considered example). At the point $N$ (intersection with the slow curve), it reaches the close proximity of the stable (attractive) branch of the manifold (relative rates of the processes are comparable on the slow curve) and begins to move along the slow curve towards the final point (slow stage $N O$ of the trajectory).

An application of the ILDM procedure provides us with more complex results, which demand additional non-trivial analysis even in the simple $2 \mathrm{D}$ case. A presence of two different eigenvectors leads to the determination of two curves, $L_{1}$ and $L_{2}$,

$$
\begin{aligned}
& L_{1}:\left(J_{11}-\lambda_{1}\right) F(\theta, \eta)+\gamma J_{12} G(\theta, \eta)=0, \\
& L_{2}:\left(J_{11}-\lambda_{2}\right) F(\theta, \eta)+\gamma J_{12} G(\theta, \eta)=0,
\end{aligned}
$$

which are built on the basis of the large negative eigenvalue (with respect to its absolute value) and the corresponding second eigenvalue of the Jacobian, respectively $\left(J_{i j}\right.$ - elements of the Jacobian of the system (5.4.1)-(5.4.2)). The curve $L_{1}$ contains branches $A B$ and $E F$, whereas the curve $L_{2}$ consists of the branches $B C$ and $F G$ (Fig. 2(b)). As it was shown (Goldfarb et al., 2004), only branches $A B$ and $F G$ have physical sense, they approximate the attractive and repulsive branches of the real manifold (compare with the $A T$ and $T V$ of the slow curve). To exclude the curves $B C$ and $E F$ from the consideration, we need to develop and apply some criteria for their disqualification. Moreover, within the band bounded by the curves $P B N$ and $U F W$, the eigenvalues of the Jacobian are complex, there is no splitting (according to ILDM!) between the two processes involved and the ILDM procedure is not applicable. As it was mentioned in Goldfarb et al. (2004), the band contains the turning point of the slow curve of the original system (5.4.1)-(5.4.2).

\subsection{Ignition of spray (two reactants model)}

Here, we consider a mathematical model of the ignition of the cool fuel spray in the hot combustible mixture. A formulation of the physical model and detailed asymptotic analysis of the dynamics of the corresponding system of the governing equations along the lines of the MIM approach can be found in Bykov et al. (2002). The finally reduced system of ODEs contains two equations and reads

$$
\begin{gathered}
\gamma \frac{\mathrm{d} u}{\mathrm{~d} \tau}=\eta(u, r)^{a} \xi(u, r)^{b} \exp \left(\frac{u}{1+\beta u}\right)-\varepsilon_{1} r\left(u+\theta_{0}\right)=F_{2}(u, r), \quad u(0)=0, \\
\varepsilon_{2} \frac{\mathrm{d}\left(r^{3}\right)}{\mathrm{d} \tau}=-\varepsilon_{1} r\left(u+\theta_{0}\right), \quad r(0)=1, \\
\eta(u, r)=\eta_{0}-\frac{\gamma u-\varepsilon_{2}\left(\nu_{\mathrm{f}} \psi-1\right)\left(1-r^{3}\right)}{\nu_{\mathrm{f}}}, \quad \xi(u, r)=1-\frac{\gamma u+\varepsilon_{2}\left(1-r^{3}\right)}{\nu_{\mathrm{o}}} .
\end{gathered}
$$

Here, $u$ is a dimensionless temperature, $\eta$ and $\xi$ are dimensionless concentrations of the fuel and oxidizer, respectively, $r$ is droplet radius, $\gamma$ is a reciprocal of the dimensionless adiabatic temperature rise and $\beta$ is a dimensionless initial temperature. The parameters $\gamma$ and $\beta$ are normally small with respect to 
unity. The parameter $\gamma$ serves as the singular parameter of the system. The value of the parameter $\varepsilon_{2}$ can essentially vary and it can serve as a singular parameter too. Therefore, the system (5.5.1)-(5.5.2) can be treated as a multi-scale system. Unlike Semenov's model, the system (5.5.1)-(5.5.2) has two small parameters and the relation fast-slow depends on the value of the ratio $\gamma / \varepsilon_{2}$. For our purposes, let us restrict ourselves by the case of the fast temperature $\left(\gamma / \varepsilon_{2} \ll 1\right)$. Assuming this, the slow curve $\Omega_{2}$ of the system (5.5.1)-(5.5.2) is given (Gol'dshtein \& Sobolev, 1992) by

$$
F_{2}(u, r) \equiv \eta(u, r)^{a} \xi(u, r)^{b} \exp \left(\frac{u}{1+\beta u}\right)-\varepsilon_{1} r\left(u+\theta_{0}\right)=0,
$$

where the chosen system parameters for the example presented here look as follows: $\beta=0.04, \gamma=$ $0.001, \varepsilon_{1}=1, \varepsilon_{2}=0.1, \psi=15, v_{\mathrm{f}}=0.127, v_{\mathrm{o}}=0.327, a=0.25, b=1.5, \theta_{0}=1.5$ and $\eta_{0}=0.25$. The slow curve $\Omega_{2}$ can be readily obtained by solving the equation $F_{2}(u, r)=0$, whereas the equations for the ILDM as well as for the TILDM are solved numerically using the continuation method ALCON (Deuflhard et al., 1987; see Fig. 3(c,d)).

It is worthwhile to follow the order of the analysis, which was adopted for the study of the Semenov model. Figure 3(a) presents the TILDM (bold dashed line), the slow curve $\Omega_{2}$ (thin solid line) and a sample numerical trajectory starting from $I$ (IABDC, the bold dotted line). Similar to the previous case, the TILDM and the slow curve almost coincide. One can readily see that the trajectory starts at the point $I(0,1)$ and moves fast towards the attractive branch $A T$ of the manifold. During this first fast stage, the slow variable $(r)$ preserves its initial value $(r=1)$. At the intersection point $A$, the relative rates of the processes involved become comparable and the trajectory begins its slow stage $(A B$, movement along the slow curve). In the vicinity of the turning point $T$, the stable branch of the slow curve loses its main feature to attract the trajectories, the trajectory tears off and the second fast stage begins ( $B D C$, final thermal explosion).

As in the previous example, the ILDM technique yields a much more rich picture, which should be additionally analysed (Fig. 3(b)). For our purposes, we depict two curves built on the basis of the two eigenvalues of the Jacobian. The curve $A B G R$ corresponds to the smaller eigenvalue $\lambda_{1}$, whereas the curve $F J K$ - to the second one $\left(\lambda_{2}\right)$. The part $A B$ of the ILDM approximates the stable branch of the slow curve/TILDM and, in fact, represents the single desired result of the ILDM machinery application. To understand the whole picture, we need to study a behaviour of Jacobian's eigenvalues. The curve GEF distinguishes the regions of the negative (left) and positive (right) values of the $\lambda_{1}$ ( $\lambda_{2}$ is positive in the chosen region of the variables values); therefore, the ILDM technique is absolutely not applicable right to the curve GEF. Moreover, the ILDM machinery demands an existence of splitting of the processes involved (essential difference in rates of changes). The curve $V W$ presents the set of points where the ratio $\left|\lambda_{1} / \lambda_{2}\right|=10$ is valid. We can see that left to the curve $V W$ the ILDM manifold approximates the slow curve quite well, whereas right to this curve the ILDM $(A B G R)$ begins to move away from the real trajectory $(A B D C)$. It can be explained by the fact that the relation between the eigenvalues decreases from 10 (on the curve $V W$ ) to the region of unity and less and this makes the application of the ILDM technique invalid.

Additional information on the dynamical picture of the system (5.5.1)-(5.5.2) can be obtained from Fig 3(c) and (d), which present both the relevant curves and the trajectories. Figure 3(c) depicts the slow curve $\Omega_{2}$ (solid line ABTF), TILDM (circles $A B T F$ ), ILDM (solid lines $A B G G$ and $K J F$ ), nuances of the grey colours correspond to different values of the Jacobian eigenvalue $\lambda_{1}$. One can easily see that the branch $A B$ is really attractive and the trajectories are attracted to it, whereas the branch $T F$ of the slow curve/TILDM is repulsive and the trajectories are rejected from it. Moreover, as we saw 
(a) Dimensionless temperature $\mathrm{u}$

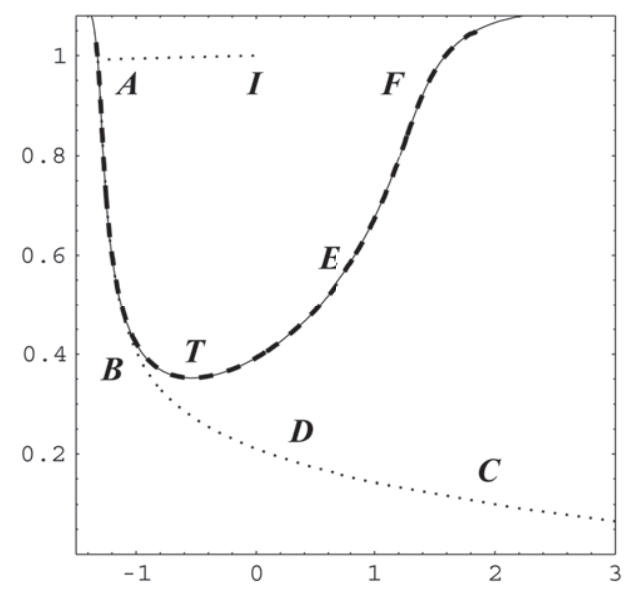

(c)

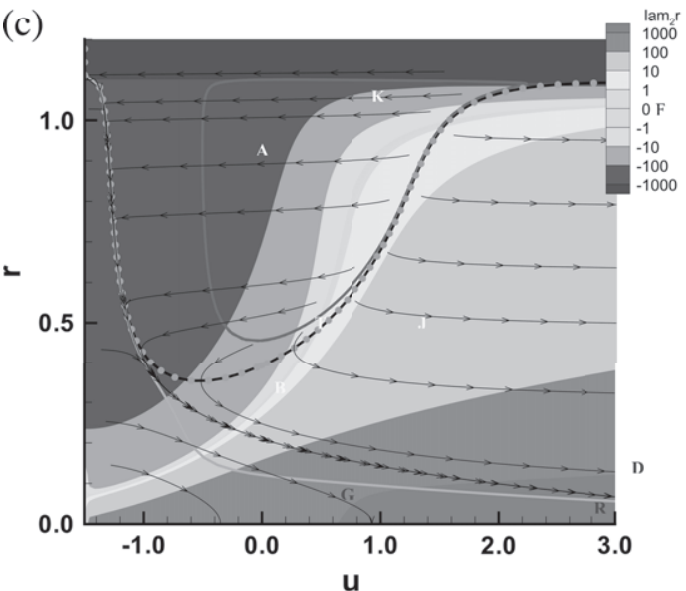

(b) Dimensionless temperature $\mathrm{u}$
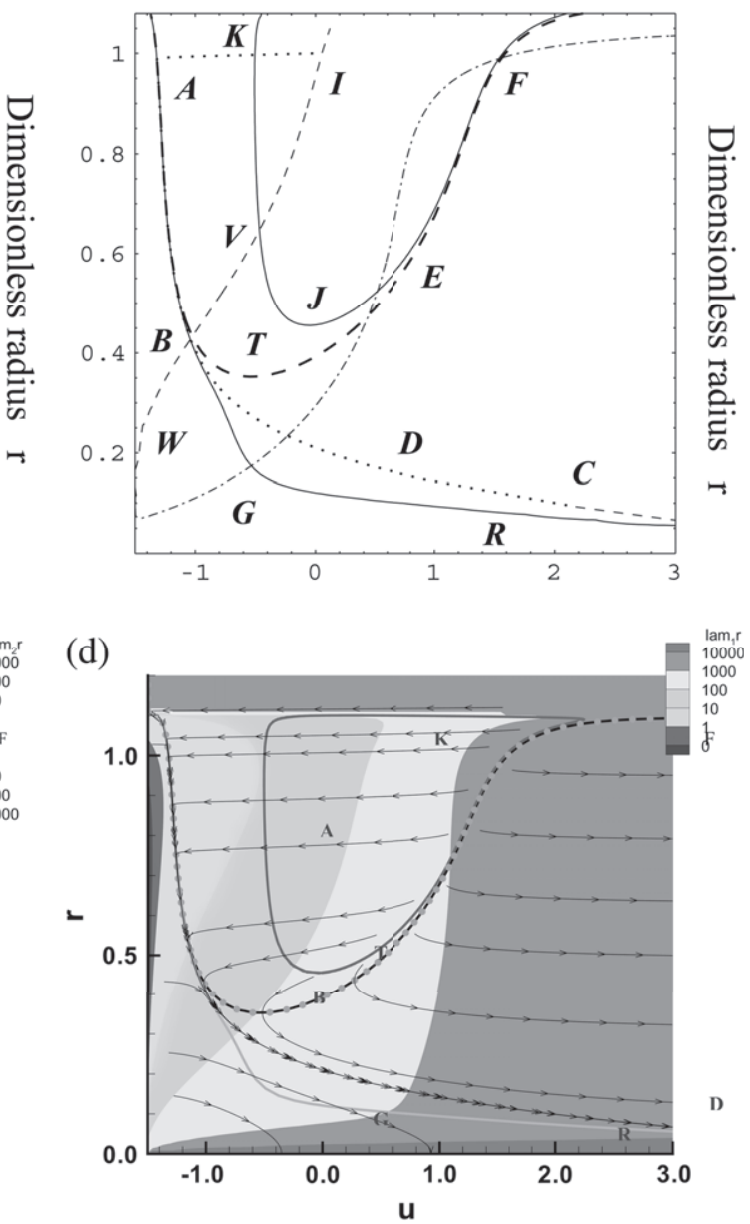

FIG. 3. The characteristic curves and the typical trajectories of the system (5.5.1)-(5.5.2) in the state plane $u-r$. (a) Slow curve (thin solid curve), TILDM (bold dashed line) and sample numerical trajectory (dotted line); (b) slow curve (dashed line), results of application of the ILDM procedure (solid lines) and sample numerical trajectory (dotted line); (c) and (d) slow curve (solid line), results of application of the TILDM procedure (circles), results of application of the ILDM procedure (solid lines) and trajectories (arrows), colours demonstrate the value of the eigenvalues $\lambda_{1}$ and $\lambda_{2}$, correspondingly.

in Fig. 3(b), in the region where $\lambda_{1}$ becomes positive, the curve $B G R$ losses its major property stops to have sense.

\section{Conclusions}

The present paper represents a natural continuation of the authors' work on a comparative analysis of the two powerful asymptotic methods-ILDM and MIM. The two are based on the general theory of integral manifolds and exploit their specific properties. Both the approaches have their own advantages and drawbacks. The complementary properties of the two asymptotic approaches (Goldfarb et al., 2004) 
and their ideological closeness generated an idea that there is a feasible combination of the two methods. Theoretically, this combination should be able to combine the advantages of these two approaches and to avoid their weaknesses. Once we develop this machinery, it can sufficiently improve both analytical and numerical study of any kind of multi-scale systems. The development of the tool is a subject of our collaborative work and the present paper is one more step in this direction.

ILDM was originally constructed to numerically handle systems of ODEs containing a large number of equations. The performed analysis showed that the ILDM method can be treated as a particular case of a general approach, which in fact has the same aim: to find a new coordinate system, where the fast and the slow motions are performed along the new axes. This all-purpose approach is formulated in the form of some type of a universal framework for all algorithms of similar nature.

As every other algorithm, ILDM has its own restrictions, which were partly demonstrated in the present paper on a number of elementary examples. It was shown that ILDM cannot treat the regions of the phase space, where the eigenvalues of the Jacobian are complex (their real values are equal and there is no splitting in rates of change of the processes involved). To overcome the discovered underwater stones, we suggested the modification of the existed ILDM method, which can be conditionally called TILDM. In the geometrical language, it represents an effective tool for numerical determination of a new system of coordinates (where the directions of the fast and slow motions coincide with or are close to the new coordinates), where the treated system of ODEs is reduced to the conventional form of the SPS. The creation of the new coordinate system is based on the local determination of the fast and slow directions (in the new desired metrics, these directions should serve as coordinates). We suggest to use a product $J J^{*}$ as the transformation matrix $M$.

The suggested approach (TILDM) was developed to identify the slow low-dimensional invariant manifolds in problems arising in mathematical theory of combustion. The distinguishing feature of this algorithm is its numerical nature and its ability to deal with large systems of ODEs (similar to ILDM). On the other hand, the results of the TILDM are close to the zeroth approximation of the exact manifold (similar to MIM).

The suggested TILDM algorithm successfully works with the systems having an internal hierarchy in the regions of the complex Jacobian's eigenvalues with close real parts. This is due to the simple fact that the new transformation matrix is symmetric and has no complex eigenvalues. In these regions, the ILDM algorithm is not applicable (there is no splitting-according to ILDM!), whereas the original system can have subdivision into fast-slow subsystems.

In the examples considered in the present paper, the suggested TILDM technique has no difficulties in the so-called transition zones (turning manifolds) between different invariant manifolds, which cannot be correctly described by the ILDM method because for these zones a division into 'large' and 'small' eigenvalues of Jacobian typically does not exist. It is also concluded that the TILDM algorithm is more sensitive to the 'hidden' hierarchy of the original system than the ILDM.

The work on the comparative analysis of the original algorithm (ILDM) and its proposed modification (TILDM) being applied to the realistic combustion problems is currently in progress. Preliminary results of this study provide us with a confidence that the proposed modification of the existing algorithm is able to overcome some of the drawbacks of its original. The authors are continuing their investigation in this direction.

Finally, we note that although the TILDM algorithm suggested here does provide a more accurate dynamical picture than that previously obtained with the ILDM, it is not without its own deficiencies (see, e.g. remark at the end of Section 4). It is undisputable that a more thorough theoretical study of the suggested approach should be performed as well as scrutinized applications to other real world problems. This direction of further improvement of suggested approach is currently under investigation. 


\section{Acknowledgements}

This research was partly supported by the Grant G-695-15.10/2001 from the GIF, the German-Israeli Foundation for Scientific Research and Development, and by the Deutsche Forschungs gemeinschaft within the Sonder Forschungsbereichs 412 and 606. IG is thankful to the European Science Foundation for its support of his visit to Germany, when this work was started. Financial support of VB by the Minerva Fellowships Program of Max Plant Society is gratefully acknowledged.

\section{REFERENCES}

Babushok, V. I. \& Gol'Dshtein, V. M. (1988) Structure of the thermal explosion limit. Combust. Flame, 72, 221-226.

Bogolyubov, N. N. \& Mitropolsky, Yu. A. (1961) Asymptotic Methods in the Theory of Nonlinear Oscillations. New York, NY: Gordon and Breach.

Bykov, V., Goldfarb, I., Gol'Dshtein, V. \& Greenberg, B. (2002) Thermal explosion in a hot gas mixture with fuel droplets: a two reaction model. Combust. Theory Model., 6, 339-359.

DAVIS, M. \& SKODJE, R. (1999) Geometric investigation of low-dimensional manifolds in systems approaching equilibrium. J. Chem. Phys., 111, 859-874.

Deuflhard, P., Fiedler, B. \& Kunkel, P. (1987) Efficient numerical pathfollowing beyond critical points. SIAM J. Numer. Anal., 24, 912-927.

FEnichel, N. (1979) Geometric singular perturbation theory for ordinary differential equations. J. Differ. Equ., 31, 53-98.

Frank-KamenetskiI, D. A. (1969) Diffusion and Heat Exchange in Chemical Kinetics, 2nd edn. New York: Plenum Press.

Goldfarb, I., Gol'dshtein, V. \& Kuzmenko, G. (1999) Pressure driven flame in porous media. Phys. Lett. A, 251, 394-403.

Goldfarb, I., Gol'dshtein, V., Kuzmenko, G. \& Greenberg, J. B. (1997) Monodisperse spray effects on thermal explosion in a gas HTD-352. Proc. ASME Heat Transf. Div., 2, 199-206.

Goldfarb, I., Gol'dshtein, V., Kuzmenko, G. \& Greenberg, J. B. (1998) On thermal explosion of a cool spray in a hot gas. Proc. Combust. Inst., 27, 2367-2371.

Goldfarb, I., Gol'Dshtein, V. \& MAAs, U. (2004) Comparative analysis of two asymptotical approaches based on integral manifolds. IMA J. Appl. Math., 69, 353-374.

Goldfarb, I., Gol'Dshtein, V., Shreiber, I. \& Zinoviev, A. (1996) Liquid drop effects on self-ignition of combustible gas. Proc. Combust. Inst., 26, 1557-1563.

Gol’dshtein, V. \& Sobolev, V. (1988) Qualitative Analysis of Singularly Perturbed Systems. Novosibirsk: Institute of Mathematics, Siberian Branch of USSR Academy of Science (in Russian).

Gol'dshtein, V. \& Sobolev, V. (1992) Singularity theory and some problems of functional analysis. AMS Transl., Ser. 2, 153, 73-92.

Gol'dshtein, V., Zinoviev, A., Sobolev, V. \& Shchepakina, E. (1996) Criterion for thermal explosion with reactant consumption in a dusty gas. Proc. R. Soc. Lond. A, 452, 2103-2119.

Hadjinicolaou, M. \& Goussis, D. M. (1999) Asymptotic solutions of stiff PDEs with the CSP method: the reaction diffusion equation. SIAM J. Sci. Comput., 20, 781-910.

HaLe, J. (1969) Ordinary Differential Equations. New York: Wiley.

KAPER, H. G. \& KAPER, T. J. (2001) Asymptotic Analysis of Two Reduction Methods for Systems of Chemical Reactions. Argonne National Lab, preprint ANL/MCS-P912-1001.

LAidler, K. J. \& Bunting, P. S. (1973) The Chemical Kinetics of Enzyme Action, 2nd edn. Oxford: Clarendon.

LAM, S. H. \& Goussis, D. M. (1988) Understanding complex chemical kinetics with computational singular perturbation. Proc. Combust. Inst., 22, 931-941.

LAM, S. H. \& Goussis, D. M. (1994) The GSP method for simplifying kinetics. Int. J. Chem. Kinet., 26, 461-486. 
MaAs, U. (1994) Automatische Rduktion von Reaktions mechanismen zur Zimulation reacktiver snomungen. Hasilitation Thesis, Universitat Stuttgart, Germany.

MAAs, U. \& Pope, S. B. (1992) Simplifying chemical kinetics: intrinsic low-dimensional manifolds in composition space. Combust. Flame, 117, 99-116.

Mcintosh, A. C., Gol'dshtein, V., Goldfarb, I. \& Zinoviev, A. (1998) Thermal explosion in a combustible gas containing fuel droplets. Combust. Theory Model., 2, 153-165.

MitropolskiY, YU. A. \& LYKOVA, O. B. (1968) Lectures on the methods of integral manifolds. Kiev, USSR: Institute of Mathematics, Ukrainen Akademy of Science, 416 p. (in Russian).

Rhodes, C., Morari, M. \& Wiggins, S. (1999) Identification of the low order manifolds: validating the algorithm of Maas and Pope. Chaos, 9, 108-123.

Roussel, M. R. (1997) Forced-convergence iterative schemes for the approximation of invariant manifolds. J. Math. Chem., 21, 385-393.

RousSEL, M. R. \& FRASER, S. J. (2001) Invariant manifold methods for metabolic model reduction. Chaos, 11, 196-206.

Semenov, N. N. (1928) Zur Theorie des Verbrennungsprozesses. Z. Phys., 48, 571-581.

Strygin, B. B. \& Sobolev, V. A. (1988) Decomposition of Motions by the Integral Manifolds Method. Moscow: Nauka (in Russian).

VALORAnI, M. \& Goussis, D. M. (2001) Explicit time-scale splitting algorithm for stiff problems: auto-ignition of gaseous mixtures behind a steady shock. J. Comput. Phys., 169, 44-79. 